\title{
Italique
}

Poésie italienne de la Renaissance

XXI | 2018

Poesia e Religione

\section{Uno scambio di sonetti tra il Lasca e Benedetto Varchi (e un omaggio a Luigi Alamanni)}

\section{Alessandro Mongatti}

\section{(2) OpenEdition}

1 Journals

\section{Edizione digitale}

URL: http://journals.openedition.org/italique/666

DOI: $10.4000 /$ italique.666

ISSN: 1663-4438

\section{Editore}

Librairie Droz

\section{Edizione cartacea}

Data di pubblicazione: 1 novembre 2018

Paginazione: 235-246

ISBN: 978-2-600-05924-4

ISSN: 1423-3983

\section{Notizia bibliografica digitale}

Alessandro Mongatti, «Uno scambio di sonetti tra il Lasca e Benedetto Varchi (e un omaggio a Luigi Alamanni)», Italique [Online], XXI | 2018, online dal 01 novembre 2020, consultato il 25 janvier 2021. URL: http://journals.openedition.org/italique/666 ; DOI: https://doi.org/10.4000/italique.666 
Aless ANDRO MONGATT I

U N O S C A M B I O D I S O N E T T I

TRA IL LASCA E B ENEDETTO VARCH I

(E U N O A G G I A L U I G A L A M A N N I) 



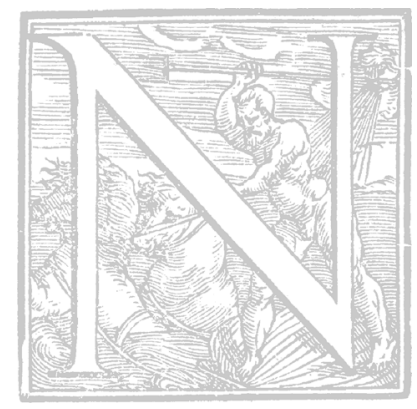

el I557 viene stampata a Firenze per Torrentino la raccolta De sonetti di $\mathrm{m}$. Benedetto Varchi colle risposte, e proposte di diversi, che l'autore intende come la Parte seconda (cosi nel frontespizio) di un'operazione unitaria, che coinvolge il volume De sonetti di m. Benedetto Varchi, Parte prima uscito nel I5s5 sempre per Torrentino ${ }^{\mathrm{I}}$ e poi, nello stesso anno, per Plinio Pietrasanta a Venezia. ${ }^{2}$ La silloge del I557 è un «epistolario poetico», ${ }^{3}$ composto da sonetti di proposta seguiti, nella stessa pagina, da quelli di risposta, divisi in due sezioni separate da una carta bianca: nella prima sono raccolti 79 scambi in cui il proponente è Varchi, nella seconda I40 nei quali Varchi risponde alle sollecitazioni di altri. ${ }^{4}$

In questa seconda sezione, a pagina 98, leggiamo il sonetto Spoglian le piagge l'herbe, e l'herbe i fiori di Antonfrancesco Grazzini detto il Lasca e la risposta varchiana Per me non hanno i prati herbe, né fiori:

(Antonfrancesco Grazzini detto il Lasca)

(Benedetto Varchi)

Spoglian le piagge l'herbe, e l'herbe i fiori, Languidi sono i gigli e le viole,

Per me non hanno i prati herbe né fiori, Lappole e stecchi son gigli e viole, Lieto non più, né chiaro, come suole, Per me sol toglie, e non dà, come suole, Rende Arno al gran Tirren suoi dritti honori. Al mondo Febo i suoi graditi honori. Piangon le ninfe, dolgonsi i pastori Odio le ninfe tutte, odio i pastori, Con sospir gravi e con meste parole, Le mie non curo, né l'altrui parole, Tanto a ciascun quaggiù rincresce e duole, Sol mi rincresce, Elpin pietoso, e duole Glorïoso pastor, de' tuoi dolori. Ma sopra tutto, oimé, la bella Flora Che più forte sono io, che i miei dolori. E credo ben, se la mia bella Flora Udisse un pur de' miei tanti sospiri, Che 'n parte cangiaria sua fronte lieta. Piange e sospira, e tra 'l pianto e' sospiri Dice, rivolta al ciel la fronte lieta: $\begin{array}{ll}\text { «Scaccia il duol, prego, omai del petto fuora } & \text { Deh, perché spiro ancor? Perché non fuora } \\ \text { Al buon Damone, e con dolci desiri } & \text { Di vita sono? O Giove i miei desiri }\end{array}$ Rendimi, o Giove, il mio maggior poeta». Adempi, e tu gli canta, almo poeta.

Questa coppia di sonetti chinde la sequenza di sei scambi tra il Lasca (proponente) e il Varchi (che risponde) cominciata a pagina 93. Nella seriazione scelta da Varchi i dodici sonettis si distribuiscono in gruppi, ciascuno composto da una coppia di scambi (ovvero quattro sonetti). 
Nei primi due scambi (pp. 93-94) il Lasca loda la virtù e la poesia di Varchi, che invita a non lasciarsi abbattere dai colpi della fortuna (Se disio sempre di fama e d'honore, p. 93) o esalta come destinato ad essere presto associato a Dante e Petrarca nel tondo dei poeti fiorentini (L'alte vigilie e gl'honesti sudori, p. 94). Varchi risponde rassicurando l'amico sulla sua intenzione di mantenersi saldo in un comportamento virtuoso nonostante le offese del «vile stuol che sempre intese / procacciar solo a' buon danno e disnore» (Grazin giusta pietà, ma troppo amore, $p .93, v v .7-8$ ) e respinge le lodi iperboliche dell' amico giustificandole come frutto di «troppo amon» (L'alte lodi, che voi del dritto fuori, $p .94, v .2$ ). I sonetti laschiani delle pp. 95-96 (rispettivamente Come è, Varchi, di nuovo in voi risorto e Tempo è omai, poi che cangiate il pelo) rimproverano invece Varchi perché, in tarda età, un nuovo innamoramento lo distrae dalla cura della propria anima e dalla preoccupazione per la vita eterna, mentre Varchi respinge l'addebito, affermando di non aver mai mutato sentimenti, che anzi l'amore l'ha sempre accompagnato per tutta la vita e che sarà proprio in virtù di esso che, neoplatonicamente, potrà ascendere a Dio (La fiamma ch'io portai nel cuore e porto $e$ Se bene io cangio d'hora in hora il pelo). I due scambi delle pp. 97-98, infine, sono accomunati dal sottogenere pastorale (a cominciare dai nomi adottati: a "Varchi》 e "Grazin» dei sonetti precedenti si sostituiscono, rispettivamente, "Damone» e «Elpin») e dalla dialettica lutto ${ }^{6} /$ consolazione, con il Lasca-Elpino che tenta di arginare la disperazione di VarchiDamone e questi che difende la propria sofferenza, invocando anzi una morte liberatrice.

In Spoglian le piagge l'herbe, e l'herbe i fiori il Lasca tratteggia un quadro di desolazione universale, con gli elementi naturali e i tipici rappresentanti del mondo bucolico (ninfe e pastori) che partecipano dei dolori del «Glorioso pastor» (v. 8), tanto che «la bella Flora», personificazione di Firenze, anche lei devastata da pianto e sospiri, prega Giove di riaccendere in Damone la dolcezza dei desideri, cosi da restituirlo alla vita e al suo ruolo di massimo poeta della città.

Nella descrizione del dolore generale che occupa $i$ primi versi il sonetto cita le Egloghe di Luigi Alamanni. ${ }^{7}$ In particolare, a prestare la maggior parte delle immagini sono alcune di quelle dominate dalla tematica funebre: la II e, come vedremo, soprattutto la III, nelle quali $i$ pastori protagonisti compiangono l'amico scomparso Cosimo Rucellai, 
UNO SCAMBIO DI SONETTI TRA IL LASCA E BENEDETTO VARCHI

con un'incursione nella $X$, dedicata alla morte del mitico Adone. Vediamo i raffronti:

Spoglian le piagge l'berbe, e l'herbe i fiori,

Languidi sono i gigli e le viole

(Grazzini, Spoglian le piagge l'herbe, e l'berbe i fiori, vv. I-2)

Voi vaghe herbette et voi già liete piante,

homai triste spogliate i fiori e 'l verde,

pallide sian le rose [...]

(Alamanni, Egloghe II 8-ıо)

[...] i fior vedove l'herbe

lasciaro, et l'herbe poi nude le piagge.

(Alamanni, Egloghe III 7 I-72).

Il lutto colpisce il mondo vegetale, in forme che il Lasca descrive secondo un'anticlimax: $i$ campi si riducono senza piante, se queste resistono non producono fiori, e i fiori che nonostante tutto si trovano mostrano segni di sofferenza ("languidi》 descrive il piegarsi dei fiori sullo stelo, ma anche la perdita di colore, esplicita nella citazione dalla II egloga alamanniana).

Lieto non più, né chiaro, come suole,

Rende Arno al gran Tirren suoi dritti honori.

(Grazzini, Spoglian le piagge l'herbe, e l'herbe i fiori, vv. 3-4)

Arno per non veder sì duro scempio

L'antico suo viaggio in dietro torse;

[...] e 'l gran Tyrrbeno un tempo

L'usato suo tributo in darno chiese.

(Alamanni, Egloghe III 64-65 e 67-68)

I due passi descrivono la stessa paradossale situazione: l'Arno per il gran dolore che patisce è impossibilitato a compiere il suo abituale «dovere» di emissario verso il mar Tirreno. ${ }^{8}$ Nel Lasca il fiume non è più «chiaro» (cioè «trasparente»), aggettivo che Alamanni gli attribuisce pochi versi dopo nella stessa egloga ("Et tu chiaro Arno al già lasciato corso», v. 92, sia pure col significato di «illustre»; e «Che più chiare d'Alpheo fian l'onde d'Arno», v. 106). 
Piangon le ninfe, dolgonsi i pastori ${ }^{9}$

(Grazzini, Spoglian le piagge l'herbe, e l'herbe i fiori, v. 5)

Le Nymphe allor ne' più diserti campi

Fuggir piangendo [...]

(Alamanni, Egloghe III 69-70)

$\mathrm{Al}$ morto giovinetto intorno piange

Il suo più fido can, piangan le Nymphe, ${ }^{\text {IO }}$

(Alamanni, Egloghe X 31-32)

Dopo gli elementi del contesto naturale e paesaggistico (le campagne, il fume), il Lasca cita i personaggi tipici del mondo bucolico, pastori e ninfe, sempre utilizzando la raccolta di egloghe di Alamanni come ipotesto.

Ma sopra tutto, oimé, la bella Flora

Piange e sospira, e tra 'l pianto e' sospiri

Dice, rivolta al ciel la fronte lieta

(Grazzini, Spoglian le piagge l'herbe, e l'herbe i fori, vv. 9-I I)

Ben vedeste dal ciel che lunghi pianti

Et che caldi sospir tutta smarrita

Sparse al vostro partir la bella Flora?

Ella stringendo a sé le dolci spoglie

Dicea con alte voci: «O stelle crude,

Crudo ciel [...]»

Così (lassa) piangea la bella Flora.

(Alamanni, Egloghe III 42-47 e 63)

Anche la partecipazione al dolore di Flora-Firenze è ripresa dalla III egloga, cosi come il suo appello in discorso diretto al cielo. Il sintagma "bella Flora», in fine di verso, è comunissimo nella poesia di Alamanni, e nella stessa egloga compare, oltre che nelle due occorrenze appena citate, anche al v. 89 ("Se qui dunque gli amasti, o bella Flora»).

A questo accorato invito di Elpino-Lasca a deporre il dolore, che coinvolge l'intero contesto naturale e la stessa Flora-Firenze con i suoi abitanti (ninfe e pastori), e che riscrive soprattutto la III delle egloghe di Alamanni, Damone-Varchi risponde rivendicando la propria disperazione e invocando la fine della vita. La replica al componimento del Lasca è architettata riprendendolo non solo per le rime, come dettavano 
UNO SCAMBIO DI SONETTI TRA IL LASCA E BENEDETTO VARCHI

le regole degli scambi di sonetti, ma per le parole, "I e addirittura - ed è in questo che risiede l'interesse dell'operazione - riutilizzando la stessa fonte.

L'incipit del sonetto di Varchi («Per me non hanno i prati herbe né fiori, / Lappole e stecchi son gigli e viole»), infatti, più che un ricordo di Petrarca, Rerum vulgarium fragmenta $166^{12}$ («altro pianeta / conven ch'i' segua, et del mio campo mieta / lappole et stecchi co la falce adunca», vv. 6-8), è in questo caso piuttosto un'allusione proprio alla III egloga di Alamanni, che nei versi 73-74 (immediatamente successivi ai passi allusi nel sonetto laschiano) recita:

Ove fur pria Narcisi, ove Hyacinti

Surgon lappole et stecchi.

Lo dimostra non solo il contesto (Varchi prende a citare la III egloga dal punto in cui si era interrotto il Lasca), ma il significato stesso della metafora «lappole e stecchi», che in Petrarca aveva un'implicazione metaletteraria (alludeva alla scelta da parte del poeta del genere lirico amoroso con la rinuncia alla Musa latina), laddove in Alamanni e poi in Varchi indica una condizione di disperata negatività. Inoltre, mentre Alamanni ricorre altre volte all'espressione, ${ }^{ }{ }^{3}$ a quel che mi consta in Varchi è l'unica occorrenza, ${ }^{\mathrm{I}}$ motivata, appunto, dall'intento di richiamare il passo alamanniano. ${ }^{\mathrm{IS}}$

Insomma, Grazzini propone a Varchi un sonetto dal «sapore» alamanniano. Varchi se ne avvede e rilancia, segnalando sin dall'incipit al proponente di aver compreso. ${ }^{16}$

D'altra parte, la figura di Luigi Alamanni doveva tornare spesso nella conversazione dei due poeti. ${ }^{17}$ In particolare, le sue Egloghe erano un riferimento per entrambi, a lungo cultori di poesia pastorale. Varchi le ricorda ancora nell'Hercolano. ${ }^{18}$ Il Lasca, poi, le elegge a modello per la propria bucolica a tutti i livelli, dall'organizzazione del macrotesto, alla strutturazione di singoli componimenti (per calco, allusione o rovesciamento), al riadattamento di immagini, motivi tessere linguistiche. Allorché, in tarda età, il Lasca decise di raccogliere tutta la propria produzione di argomento bucolico per stamparla in più libri di Opere pastorali, ${ }^{19}$ subito dopo le egloghe collocò una serie di 62 sonetti ${ }^{20}$ che la rubrica del primo componimento dedica "Al pastore Alfesibeo inteso per Luigi Alamanni», ${ }^{2 \mathrm{I}}$ quale riconoscimento dell'impronta lasciata dalla produzione poetica di Luigi sulla pastorale di Grazzini. 
Il 27 maggio I542, quando Varchi non era ancora rientrato a Firenze dall'esilio, il Lasca, che allora non lo conosceva personalmente ma lo ammirava da lontano come una sorta di maestro ideale, gli aveva inviato a Bologna una lettera, in cui lo dichiarava suo «secondo maestro» dopo Petrarca, circoscrivendone il magistero ad alcuni settori, con al primo posto la poesia pastorale, distinta in sonetti e egloghe:

non veggo mai vostri componimenti che io non impari qualcosa; come ho fatto primieramente dai sonetti vostri pastorali, dall'egloghe, dalle traduzioni, dalle letture, ed infino dai capitoli burleschi. In fine voi siete il mio secondo maestro, già per i consigli vostri avendomi eletto il Petrarca pel primo; sicché dove io non posso imitarlo, o per dir meglio ingegnarmi, a voi ed all'opere vostre ricorro $[. ..] .{ }^{22}$

Almeno per quel che riguardava le egloghe si trattava di una piccola bugia: ${ }^{23}$ nella scrittura di egloghe Lasca guarda al modello di Luigi Alamanni, ${ }^{24}$ molto piu che a quello di Varchi. ${ }^{25}$ Dopo che questi a inizio 1543 tornò a Firenze, $i$ due ebbero modo di conoscersi bene, entrando in confidenza e diventando amici, schierandosi dalla stessa parte nelle contrapposizioni interne all'Accademia Fiorentina e rivolgendosi a lungo componimenti poetici, di stampo petrarchesco come pure (da parte del Lasca) burlesco. Mi piace pensare che, scambiandosi pareri sulle rispettive composizioni, abbiano riso assieme di quell'innocente esagerazione del Lasca e abbiano idealmente omaggiato il valore della poesia pastorale di Alamanni.

Al di là della verosimiglianza della scena, il ricorso all'ipotesto delle Egloghe nel sonetto di proposta del Lasca che abbiamo rintracciato, con la replica di Varchi sulla stessa falsariga, non è soltanto un esempio di dialogo implicito tra un poeta che chiama il proprio interlocutore a riconoscere un modello e a rispondere di conseguenza, ma è anche l'allusione a una sorta di lessico familiare, un richiamo a un comune modello poetico, un mezzo per rinsaldare la loro consonanza intellettuale.

Alessandro Mongatti 
Ringrazio per l'attenta lettura e i suggerimenti Simone Albonico, Nicoletta Marcelli, Alberto Martelli, Alessandro Parenti e Dario Pecoraro. Naturalmente errori e imprecisioni sono mia responsabilità.

I. Con dedica a «don Francesco Medici principe di Firenze».

2. Con dedica a Giovanni Della Casa firmata da Giorgio Benzoni e aggiunta di tre egloghe finali.

3. Giuliano Tanturli, Una gestazione e un parto gemellare: la prima e la seconda parte dei Sonetti di Benedetto Varchi, «Italique», VII (2004), pp. 43-100, p. 46.

4. Nella sproporzione delle due sezioni si può apprezzare lo statuto di punto di riferimento intellettuale, al centro di una fitta rete di scambi e rapporti, guadagnato negli anni da Benedetto Varchi, che decine di personalità di tutta Italia hanno interesse a coinvolgere in uno scambio poetico.

5. I precedenti sono: Se disio sempre di fama e d'honore / Grazin giusta pietà, ma troppo amore, p. 93; L'alte vigilie e gl'honesti sudori / L'alte lodi, che voi del dritto fuori, p. 94; Come è, Varchi, di nuovo in voi risorto / La fiamma ch'io portai nel cuore e porto, p. 95; Tempo è omai, poi che cangiate il pelo / Se bene io cangio d'hora in hora il pelo, p. 96; Alle lagrime triste almo pastore / Si Pale al buono Elpin sempre in migliore, p. 97.

6. Il primo sonetto (Alle lagrime triste almo pastore) di questo doppio scambio chiarisce che il dolore di Varchi è motivato dalla scomparsa di qualcuno che gli era caro, con il Lasca che lo invita a moderare la sofferenza tramite il tradizionale argomento cristiano che descrive come felice, e non dolorosa, la sorte di chi muoia in stato di grazia: «Alle lagrime triste, almo pastore, / Pon fine, e lascia il languir tuo cotanto; / Pensi tu forse che 'l soverchio pianto / Faccia da te partir stanco il dolore? / Dove è l'antico senno, ove è il valore / Che nell'altrui sventure oprava tanto? / Hor non sai tu che nel beato e santo / Regno sen va chi ben vivendo muore? / Dunque a che più dolersi? A che più fare / Grave a sé stesso et agl'amici oltraggio / Piangendo sempre indarno l'altrui bene? / Spoglia Damone, omai, spoglia l'amare / Doglie vane, e col nuovo e lieto maggio / Vesti nuova dolcezza e lieta spene». La seconda coppia di sonetti (Spoglian le piagge l'herbe, e l'herbe i fiori / Per me non banno $i$ prati herbe, né fiori, p. 98), che qui sto esaminando, priva di riferimenti concreti, potrebbe teoricamente riferirsi a una circostanza diversa, ma tendo ad escluderlo, sia per la collocazione scelta da Varchi nella sua edizione (che, come detto, raduna gli scambi con il Lasca a coppie), sia perché i due sonetti laschiani si ritrovano (privi delle risposte) associati nella stessa carta (44r) pure nel manoscritto autografo conservato alla Biblioteca Nazionale di Firenze e siglato Magl. VII i 240, nel quale, come dirò anche più avanti, il Lasca intendeva raccogliere la propria produzione pastorale.

7. Che a sua volta attinge alla poesia pastorale classica, e in particolare ai bucolici greci, da Teocrito al compianto di Mosco per Bione. Le Egloghe si leggono in Luigi Alamanni, Opere toscane al Christianissimo Re Francesco Primo, Lugduni, Gryphius, I 532 , I, pp. I08-I 87.

8. Il motivo del corso d'acqua che, sfociando nel mare, compie un proprio obbligo (di natura tributaria) è particolarmente diffuso nella poesia alamanniana: 
cfr., per limitarci alle Opere toscane, anche Alamanni, Favola di Athlante 337-338: «[...] rapidi torrenti / ch'all'Athlantico mar tributo fanno»; Alamanni, Sonetti. Prima parte I 2, I-3: «Padre Ocean, che dal gelato Arcturo / ver l'occidente i tuoi confini stendi, / et de' Gallici fiumi il dritto prendi»; Alamanni, Diluvio romano i6: «ove il Gallico mar suo dritto attende» (con riferimento alle acque del «Rhodan superbo»); Alamanni, Inni $\mathrm{V}$ 78: «rende il suo dritto al mare» (il soggetto è l'Histro, cioè il Danubio). Compare anche altrove nel Lasca: per rimanere nell'ambito della poesia pastorale, vedi la prima delle sue egloghe (composta nel i 539 per il matrimonio del duca Cosimo de' Medici con Eleonora di Toledo): «Così del mio vago Arno amate e care, / honorato Signor, vostra mercede, / andranno l'acque a dar suo dritto al mare» (Vv. I6I-I63). Pochi versi più avanti (I65-I66), si trova invece l'immagine del fiume che al mutare del proprio stato emotivo altera la velocità del corso e la trasparenza delle acque: «e più che mai sendo tranquille $\mathrm{e}$ chiare / della sua gioia e pace faran fede», presente anche in questo sonetto, nel passo citato (v. 3: «Lieto non più, né chiaro»).

9. Per l'accoppiamento, topico, cfr. almeno Ovidius, Metamorphoses VI 394-396: «et nymphae flerunt, et quisquis montibus illis / lanigerosque greges armentaque bucera pavit».

ı. Con rimando a Vergilius, Bucolica V 20-21: «Exstinctum Nymphae crudeli funere Daphnin / flebant».

I I. Come già avveniva, in questo gruppo di scambi tra Grazzini e Varchi, per quello di p. 96: Tempo è omai, poi che cangiate il pelo / Se bene io cangio d'hora in hora il pelo.

I 2. E per suo tramite di Vergilius, Georgica I i 53.

I 3. Nelle Opere Toscane, in Satire V i 5 I-I 5 2: «Che 'l mondo nel suo sen conosca certo / Quant'ha lappole et stecchi [...]» e Selve I VII 6o: «Lappole, stecchi, roghi e 'l tasso amaro».

14. Nella raccolta De sonetti di $\mathrm{m}$. Benedetto Varchi colle risposte, e proposte di diversi compare un'altra volta, alla p. 203 , ma in un sonetto non di Varchi, bensì del proponente Francesco Bolognetti, che la impiega «petrarchianamente» con significato metaletterario (vv. 9-I I: «Ben veggio io ancor, che sol lappole e stecchi / Di sì sterile campo e paglia ignuda / E felce senza frutto alcun si miete»: Bolognetti sta parlando di un suo libro, citato al v. 2, che ha inviato a Varchi su richiesta di questi). È invece leggibile nella Mascherata, la cui paternità varchiana è però dubbia: testimoniata dal codice della Biblioteca Nazionale di Firenze BNF II VIII I46 alle cc. 32v-34r, fu pubblicata nel 1837 da Giuseppe Aiazzi in Benedetto VARCHI, Saggio di rime inedite, estratte dai manoscritti originali della Biblioteca Rinucciana, Firenze, Pialli (sulla questione, vedi Salvatore Lo Re, L'amico di Benedetto: Luca Martini, in Politica e cultura nella Firenze cosimiana. Studi su Benedetto Varchi, Manziana, Vecchiarelli, 2008, pp. 257-294, specialmente pp. 274-276).

I 5. L'espressione compare invece nello stesso Lasca sempre in contesto pastorale, nella sua VIII egloga, ancora a indicare una condizione disforica, dovuta in quel caso alle pene dell'amore: «Lasso!, che nel più bel fiorito maggio / 
invece all'herbe e i fior, lappole e stecchi / nel più rio mieterai crudo dicembre» (Grazzini, Egloghe, VIII 59-6i). La datazione delle dieci egloghe grazziniane è questione ancora aperta. A parte le prime due, collegate a eventi ufficiali del i 539 e I 54I (il già citato matrimonio di Cosimo I e Eleonora di Toledo e la nascita del loro primo figlio maschio Francesco), è difficile collocare nel tempo le altre. Se l'VIII è stata scritta come le prime due tra fine anni Trenta e inizio anni Quaranta, il sonetto di Varchi opererebbe una doppia citazione, col richiamo all'ipotesto scelto dal Lasca e il contemporaneo omaggio al proprio interlocutore. Se invece l'egloga è successiva a questo scambio di sonetti, è il Lasca a citare oltre alle Egloghe di Alamanni (largamente saccheggiate in tutta l'egloga) anche la conversazione poetica intercorsa con Varchi.

I6. Varchi poteva vantare una perfetta e sicura conoscenza della lirica del suo tempo, che gli aveva guadagnato una posizione di assoluto prestigio, tanto che letterati e aspiranti poeti di tutta Italia si rivolgevano a lui per chiedergli consigli e gli sollecitavano una presa di posizione sulle frequenti dispute di poetica. Cfr. Marco Biffi - Raffaella Setti, Varchi consulente linguistico, in Benedetto Varchi. I503-I565, a cura di Vanni Bramanti, Roma, Edizioni di Storia e Letteratura, 2007, pp. 25 -67.

17. Varchi aveva incontrato più volte il più anziano Luigi, al quale era stato legato pure per la comune militanza repubblicana. Nella stessa raccolta De sonetti di m. Benedetto Varchi colle risposte, e proposte di diversi sono riportati 3 scambi di sonetti tra i due. Sono giunte sino a noi 6 lettere di Alamanni a Varchi, che testimoniano una consuetudine di frequentazioni e scambi e un'evidente stima reciproca ( $c f r$. Lettere a Benedetto Varchi (I530-I563), a cura di Vanni Bramanti, Manziana, Vecchiarelli, 201 2, lettere 60, 61, 64, 65, 68, 78).

I 8. «Oltra questi cinque modi, ce ne sono due da cantar cose pastorali, uno in burla, come la Nencia di Lorenzo de' Medici e la Beca di Luigi Pulci, e l'altro da vero, e questo si divide in due, perché alcuni scrivon l'egloghe in versi sciolti, come sono quelle di messer Luigi Alamanni e di messer Hieronimo Muzio, e di molti altri, e alcuni in versi rimati, e questo si fa medesimamente in due modi, o con rime ordinarie o con rime sdrucciole, come si vede nel Sannazzaro» (Benedetto VArchi, Hercolano, edizione critica a cura di Antonio Sorella, Pescara, Libreria Università Editrice, I995, pp. 804-805).

I 9. Il progetto, che occupò gli ultimi anni della vita del Lasca, non andò in porto, ma è testimoniato dall'autografo della Biblioteca Nazionale di Firenze Magl. VII I 240 citato alla nota 6 .

20. Tra i quali, come già detto, figurano alla c. $44 r$ anche Alle lagrime triste almo pastore e Spoglian le piagge l'herbe, e l'herbe i fiori, accolti nella stampa varchiana del I 557 .

2 I. Nel manoscritto si legge una precedente dedica a «Giovambatista di Lorenzo Strozzi», poi cancellata e sostituita con quella ad Alamanni.

22. Pubblicata in Lettere a Benedetto Varchi (I530-I563), cit., pp. 214-I6; citata in Annalisa Andreoni, La via della dottrina. Le lezioni accademiche di Benedetto Varchi, Pisa, ETS, 201 2, pp. 6r-62. 
23. Motivata da una comprensibilissima captatio benevolentiae da parte di un poeta non affermato che osava rivolgersi a un letterato di fama.

24. Chi scrive sta lavorando all'edizione critica e commentata del manoscritto magliabechiano che raccoglie le Opere pastorali del Lasca. La funzione di modello diretto delle Egloghe alamanniane su quelle del Lasca sarà puntualmente ed estesamente dimostrata in quella sede. Per un primo accenno, mi permetto di rimandare a Alessandro Mongatti, La I ecloga di Calpurnio Siculo modello di Dimmi, Damon, perché sì dolcemente di Antonfrancesco Grazzini detto il Lasca, "Studi Italiani di Filologia Classica», CIX (2016), pp. I49-I66 e ID., "La vaga et bella fiammeggiante Aurora». Un ciclo di poesie dell'Accademia degli Umidi per la nascita di Francesco I de' Medici (I54I), «Nuova Rivista di Letteratura Italiana», XX (2017), I, pp. I $4 \mathrm{I}-\mathrm{I} 74$.

25. All'altezza cronologica della lettera, Varchi ha scritto già due delle tre sue egloghe che conosciamo, entrambe risalenti all'estate del i 539: l'Amarilli, che fu inviata da Varchi, che allora si trovava a Padova, a Cosimo di Palla Rucellai nell'estate I 539, e la Dafni, che Varchi trasmise a Ugolino Martelli il io settembre I 539 (cfr. Giovanni Ferroni, Una lettera di Benedetto Varchi nel ms. Laur. Ashb. 1039, in Salvatore Lo Re, Franco Tommasi (a cura di), Varchi e altro Rinascimento. Studi offerti a Vanni Bramanti, Manziana, Vecchiarelli, 201 3, pp. 47-60). La terza, Damone, risale al I $553-$ I554. 